Der Sonntag war der Thematik des Staatsangehörigkeitsrechts gewidmet. Prof. Dr. Gerard-Rene de Groot, Universität Maastricht, führte mit einem humorvoll akzentuierten Referat zum Staatsangehörigkeitserwerb durch das „ius soli“, wie er seit dem StAG 2000 auch für Kinder ausländischer Eltern mit entsprechendem Aufenthaltsstatus in der Bundesrepublik möglich ist, in die Thematik ein. Dabei stellte der Referent rechtsvergleichend die Regelungen der 15 alten EU-Länder, Österreichs und der Schweiz dar. Einige Länder haben ein „doppeltes ius soli“ geregelt: ist bereit ein ausländischer Elternteil z.B. in Belgien, Portugal oder Frankreich geboren, erwirbt das Kind, das in diesem Land geboren wird, die Staatsangehörigkeit des Landes. Nur Deutschland hat einen Optionszwang in \29 Abs. 1 StAG geregelt. 13 von 18 Staaten akzeptieren eine mehrfache Staatsangehörigkeit, in einzelnen Ländern kann die Aufgabe der Staatsangehörigkeit schlicht erklärt werden.

Das sich anschließende provokante Thema des Podiumsgesprächs „Abschaffung des Optionszwangs“ verlangte von den Teilnehmerinnen und Teilnehmern eine Positionierung zur gegenwärtigen Rechtslage, die für die ersten jungen Erwachsenen bald Realität wird. Der Richter am BVerwG Prof. Dr. Uwe Berlit hielt den Optionszwang zwar nicht für wünschenswert, wenn nicht gar für unsinnig, aber für verfassungsrechtlich zulässig. Prof. Dr. Kay Hailbronner wies darauf hin, dass in der Praxis das Ziel der gesetzlichen Regelung - eine Vermeidung der doppelten Staatsangehörigkeit - schwer zu erreichen sei. Der Vorsitzende der türkischen Gemeinde in Deutschland Kenan Kolat nannte die Regelung und die Tatsache, dass nun insbesondere türkische junge Erwachsene optieren müssten, für einen „integrationspolitischen Scherbenhaufen“. Diesen sei nicht zu vermitteln, warum einige ihrer Freunde ihre (durch Einbürgerung erworbene) deutsche Staatsangehörigkeit ohne weiteres behielten, sie aber im Fall „vergessener (und damit nicht ausgeübter) Option “ ihre deutsche Staatsangehörigkeit verlören. Auf diese Weise schaffe man nicht nur praktische Probleme wie fehlende türkische Meldepapiere, sondern auch zwei Identitäten. Dabei müsse anerkannt werden, dass die Person auf Grund ihrer Sozialisierung in Deutschland und ihrer ausländischen Wurzeln beide Identitäten in sich vereinige. Auf dieses Phänomen verwies auch Gabriele Erpenbeck (Zentralkomitee der Deutschen Katholiken). Sybille Laurischk (MdB, FDP) verlangte, dass ein neuer Diskurs zum Staatsangehörigkeitsrecht mit dem Ziel einer möglichen Gesetzesänderung angestoßen werde.

Mit diesem übereinstimmenden Appell an den Gesetzgeber schloss eine anregende Tagung in Stuttgart-Hohenheim, die wie immer von Klaus Barwig, Dr. Gisbert Brinkmann und Dr. Christoph Schumacher durch die Gewinnung interessanter Referentinnen und Referenten sehr gut vorbereitet und souverän geleitet wurde.

\title{
GF2D/CRIFF - ein Frauenrechtsverband aus Togo
}

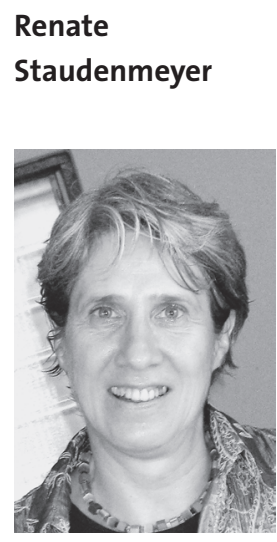

Fachkraft des DED (Deutscher Entwicklungsdienst), Lomé/Togo
Sylvia Cleff

Le Divellec, LL.M.

(Paris 2)

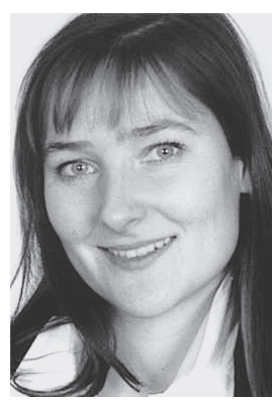

Mitglied der Kommission Öffentliches Recht, Europa- und Völkerrecht des djb, Beraterin/Juristin für die französische Unternehmensberatung „ALETEYA - conseil en diversité" in Paris
GF2D wurde 1992 als Nichtregierungsorganisation in Togo (Westafrika) von ca. 30 Juristinnen und Frauen mit unterschiedlichem beruflichem Hintergrund gegründet.

Nach der Erarbeitung einer Satzung hat sich dieser Frauenrechtsverband seit 1994 ein Exekutiv-Organ zur Seite gestellt, das „Centre de Recherche d'Information et de Formation pour la Femme“ (CRIFF). CRIFF ist damit beauftragt, diejenigen Aktivitäten umzusetzen, die in einem Fünfjahresplan für die drei Programmbereiche von GF2D konzipiert und geplant werden: für den Bereich juristische Dienstleistungen, den Bereich bürgerrechtliche Bildung von Frauen und den sozioökonomischen Bereich.

Der juristische Programmbereich von GF2D/CRIFF verfolgt die Verwirklichung übergeordneter Zielsetzungen, wie

- den Zugang von Frauen zum Recht, die Kenntnis ihrer (bürger-)rechtlichen Situation sowie deren effektive Nutzung zu maximieren,

- Frauenrechte auf nationaler Ebene allgemein verständlich zu vermitteln,

- die LaienjuristInnen so zu organisieren, dass die Wirkungen ihrer Aktivitäten durch die Verbesserung ihrer Arbeitsbedingungen optimiert werden,

- den rechtlichen Status von Frauen in Togo zu verbessern,

- geschlechtsspezifische Gewalt zu reduzieren, 
- sich für eine breiteres Engagement von Männern für Frauenrechte einzusetzen,

- Studien zu feministischen Themen in Afrika voranzubringen.

Überzeugt davon, dass die Verbesserung der rechtlichen und sozialen Situation von Frauen in Togo Schlüsselelemente darstellen, um wirkliche demokratische und nachhaltige Entwicklung im ganzen Land voranzubringen, hat GF2D/CRIFF folgende zwei Hauptbereiche in den Mittelpunkt seines Engagements gestellt:

\section{Fortbildungen im Bereich der rechtlichen Bildung von Frauen in Togo}

\section{Parajuristes/LaienjuristInnen:}

GF2D/CRIFF hat sich als erste Organisation in Togo für die Ausbildung sogenannter Laienjuristinnen eingesetzt (parajuristes). Bereits von 1994 bis 2000 wurden ca. 300 Frauen als Laienjuristinnen geschult, die man auch „les juristes aux pieds nus “ 1 nennt. Inzwischen gibt es im ganzen Land fast 600 solcher Laienjuristinnen und -juristen, die eine Fortbildung von GF2D erhalten haben und regelmäßig weiter geschult werden. Die ausgebildeten Laienjuristinnen und -juristen (seit 2001 werden auch einige Männer ausgebildet) sind in 33 regionalen Netzwerken organisiert. Mit ihrem ehrenamtlichen Engagement stehen sie gezielt Frauen, aber auch der gesamten Bevölkerung mit juristischer Aufklärung und Beratung zur Seite und sensibilisieren sie für die Bedeutung der Wahrnehmung ihrer bürgerlichen Rechte.

Ein Kernelement der Aktivitäten der LaienjuristInnen stellt die Aufklärungs- und Sensibilisierungsarbeit der Bevölkerung in ihrem Umfeld dar, die oft mit einfachem didaktischem Material begleitet wird (selbst entwickelte Sketche, Radiosendungen mit den lokalen Radiosendern, falls vorhanden Plakate oder Faltblätter in lokaler Sprache etc.).

Auf Initiative der LaienjuristInnen gibt es mittlerweile im ganzen Land sieben Beratungszentren (2 x Lomé, Tsévié, Kpalimé, Atakpamé, Bafilo und Kara). Hier werden Lösungen für juristische Problemstellungen gesucht, wenn es um familiäre Probleme geht, um Scheidung, Unterhalt der Kinder, Kinderrechte und Elternpflichten, um die Bedeutung von amtlichen Bescheinigungen bei Geburten oder Heirat, Rechtsschutz im Falle von sexueller Gewalt und sexueller Belästigung, oder wenn Witwen unter den sozial ausgrenzenden und erniedrigenden Erbrechts-Ritualen leiden (in denen die Frauen u.a. belegen müssen, dass sie nicht am Versterben des Ehemannes beteiligt waren).

Neben der ethnischen Vielfalt (über 40 verschiedene ethnische Gruppierungen) gibt es in Togo eine weitere Besonderheit, die sich auf die juristische Beratungsarbeit von GF2D/ CRIFF auswirkt: im westafrikanischen Kontext spielen nicht nur offizielle Gesetzestexte eine Rolle für die Rechtsgebung und Rechtsprechung, sondern es existieren daneben „les droits coutumiers“2, die von der jeweiligen lokalen „chefferie traditi- onnelle" umgesetzt werden und je nach Region eine mehr oder weniger große und gerade für Frauen benachteiligende Rolle spielen.

\section{Lobbying, Öffentlichkeits- und Bildungsarbeit für die Förde- rung von Frauen in öffentlichen Positionen}

GF2D gehört in Togo zu den renommierteren zivilgesellschaftlichen Organisationen und war als Vertreterorganisation von Togos Zivilgesellschaft am Zustandekommen und der Unterzeichnung des „Accord Politique Global“ im August 2006 beteiligt. In dieser Funktion hat GF2D ein kontrollierendes Auge auf Handlungen und Entscheidungen der togoischen Regierung. Dies ist gerade im Moment, da Togo eine wichtige Phase der Demokratisierung durchläuft, von enormer Bedeutung. Auch hier ein konkretes Beispiel: Im Mai 2007 wurde auf dem Projektgelände eine Pressekonferenz abgehalten, da eines der obersten Gerichtsorgane, das Verfassungsgericht, vom togoischen Parlament neu besetzt wurde - allerdings ausschließlich mit männlichen Vertretern der Regierungspartei. Im Rahmen der Pressekonferenz wurde dies von GF2D deutlich kritisiert und der Präsident direkt aufgefordert, seine Verantwortung gemäß den Vereinbarungen des abgeschlossenen „Accord Politique Global“ einzuhalten. In diesem ist unter anderem festgelegt, dass Frauen in Entscheidungspositionen beteiligt werden sollen. Folge der Pressekonferenz: immerhin wurde eine Frau unter die (männlichen) Mitglieder des „Cour constitutionnelle" berufen.

„Die Frauen selbst werden über ihre Rechte aufgeklärt und dabei unterstützt, diese auch anwenden zu können. “ - so beschreibt Mme Kafui Afiwa Kuwonu, aktuelle Vorstandsvorsitzende, den Arbeitsansatz von GF2D/CRIFF, und weiter: „Entscheidungsträger werden davon überzeugt, dass Bedingungen geschaffen werden müssen, die der Anerkennung und Wahrung von Menschen- und Frauenrechten Rechnung tragen.

Heute wissen wir, dass der Ansatz von GF2D die Bevölkerung in das Bewusstsein ihrer rechtlichen Position gebracht hat, so dass sie als Bürgerinnen und Bürger verantwortlich handeln können: Frauen erkennen ihre Rolle und ihren (rechtlichen) Platz in der Gesellschaft. Wir sind jedoch noch weit davon entfernt, dass Frauen ihre Rechte vollständig wahrnehmen. Dennoch bestärken unsere Aktivitäten die togoischen Frauen darin, ihre Rechte im familiären und lokalen Umfeld wahrzunehmen. Und es ist auch nicht zu unterschätzen, dass Organisationen der Zivilgesellschaft wie GF2D/CRIFF eine wichtige Rolle in der Begleitung und kritischen Beobachtung der Regierung einnehmen, dass sie ein Auge darauf haben, damit politische Entscheidungen zum Wohle des Volkes getroffen werden.“

\footnotetext{
1 Wörtlich übersetzt „die barfüßigen Juristen“, eine bildliche Beschreibung der LaienjuristInnen.

2 Gewohnheitsrechte.

3 Interview für DED-Brief, März 2009.
} 